\title{
ARTICLE
}

\section{Validation of the international reactor dosimetry and fusion file}

\author{
Stanislav Simakov ${ }^{\mathrm{a}^{*}}$, Lawrence Greenwood ${ }^{\mathrm{b}}$ and Roberto Capote ${ }^{\mathrm{a}}$ \\ ${ }^{a}$ International Atomic Energy Agency, Vienna, P.O. Box 100, Austria; ${ }^{b}$ Pacific Northwest National Laboratory, Richland, WA, P.O.
}

Box 999, USA

The International Reactor Dosimetry and Fusion File (IRDFF) is an extension of the International Reactor Dosimetry File (IRDF-2002) to cover fission, fusion and accelerator driven applications up to an energy of $60 \mathrm{MeV}$ and now includes 75 reactions. The Nuclear Data Section of IAEA initiates a Coordinated Research Project (CRP) with the main goal to validate and test the IRDFF library. This paper will describe the scope and present status of IRDFF as well as the objectives of the CRP, i.e. the overview of experimental data required for validation. We inform the community about this activity and draw the attention of researchers who can make a contribution.

Keywords: international collaboration; dosimetry cross sections; accelerator and reactor driven neutron sources; experimental validation

\section{Introduction}

The International Reactor Dosimetry File (IRDF-2002) [1] developed under the auspices of the Nuclear Data Section (NDS) of the IAEA was recently extended to cover additional fusion and accelerator driven applications and will be known as the International Reactor Dosimetry and Fusion File (IRDFF) [2]. The extension includes 29 new reactions and increases the end-point energy from 20 to $60 \mathrm{MeV}$. The energy extrapolation has been made in a formal way by using the TENDL-2010 cross sections and covariance matrices [4] after matching to the IRDF-2002 values at $20 \mathrm{MeV}$.

The NDS launches a Coordinated Research Project (CRP) to benchmark and test IRDFF and release it as a validated numerical database for wide use [3]. This paper describes the scope and present status of IRDFF as well as the objectives of the CRP - the overview of the experiments suitable for the validation purpose.

\section{The scope of IRDFF and validation}

The International Reactor Dosimetry and Fusion File is a standardized evaluated cross section library of neutron dosimetry reactions with uncertainty information that supersedes the widely used IRDF-2002. The IRDFF contains cross section data and related decay data for 75 dosimetry reactions, and absorption data for three cover materials B, Cd and $\mathrm{Gd}$ used in combination with some detectors.

Preliminary validation [2] has been done in three

\footnotetext{
* Corresponding author. Email: s.simakov@iaea.org
}

standard neutron fields: thermal Maxwellian, 1/E, and ${ }^{252} \mathrm{Cf}$; and one reference field: prompt fission spectrum of ${ }^{235} \mathrm{U}\left(\mathrm{n}_{\text {thermal }}, \mathrm{f}\right)$ (the latter, however, has large uncertainties at low and high emission energies). Any new and additional measurements in such standard and reference fields in accordance with ASTM guide [5] would be a valuable contribution to this CRP.

Additionally other neutron fields (the so called controlled) are often used for validation of the cross sections for specific applications and energy ranges such as: spallation, fusion (including International Fusion Material Irradiation Facility spectrum extending up to $60 \mathrm{MeV}$ ), critical and reactor facilities. For benchmarking purposes the neutron field has to be rather well characterized and documented, including detailed description of uncertainties and their correlations [5]. To reach the verification goal, the spectrum uncertainty should be less than or comparable with the cross section uncertainties under study in the range of sensitivity. The necessary level of spectrum accuracy could be achieved by the combined use of differential spectrometry, analytical calculations and integral measurements.

To facilitate the CRP goals NDS has recently held a dedicated meeting "Neutron Spectra for EXFOR" to collect relevant information for wide use [6].

\section{Validation of IRDFF by accelerator driven sources}

\subsection{High energies $(20-100 \mathrm{MeV})$}

One of the main focuses of the CRP will be a validation of the extension above $20 \mathrm{MeV}$, since it was done by reaction cross section modeling. Analysis of data 
available in EXFOR shows that there are too few measurements at energies from 20 to $100 \mathrm{MeV}$ and reported uncertainties are large. This is illustrated in Figure 1 which displays the available experimental cross sections for the ${ }^{59} \mathrm{Co}(\mathrm{n}, 3 \mathrm{n})$ reaction together with evaluations from IRDFF and EAF-2010 [7]. The spread of these scarce data and their $30-50 \%$ uncertainties definitely point out the need for new cross section measurements. For this purpose the quasi-monoenergetic ${ }^{7} \mathrm{Li}(\mathrm{p}, \mathrm{xn})$ neutron source is usually employed [8], which however also produces low energy neutrons. These neutrons can even make a dominant contribution in the case of low threshold reaction, resulting in a kind of integral experiment.

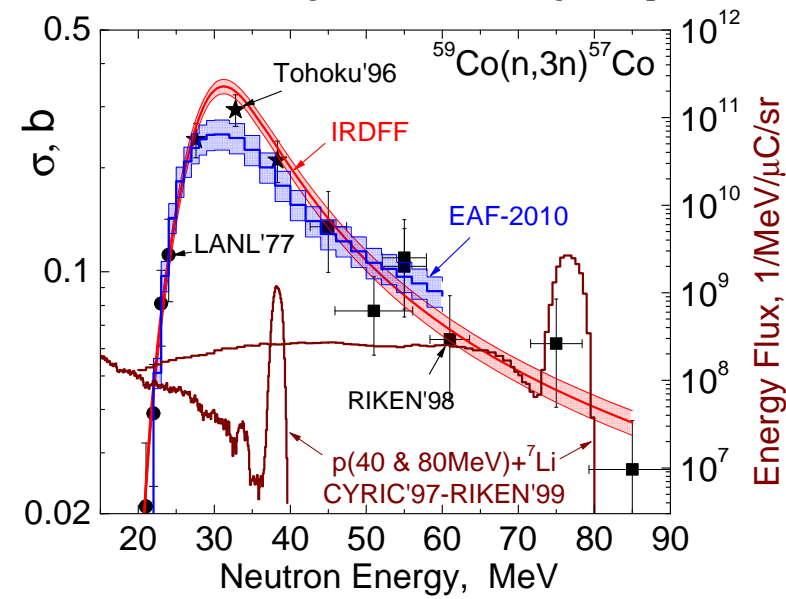

Figure 1. Experimental (EXFOR) and evaluated cross sections for ${ }^{59} \mathrm{Co}(\mathrm{n}, 3 \mathrm{n})$ reaction and $\mathrm{p}-{ }^{7} \mathrm{Li}$ source spectra [8].

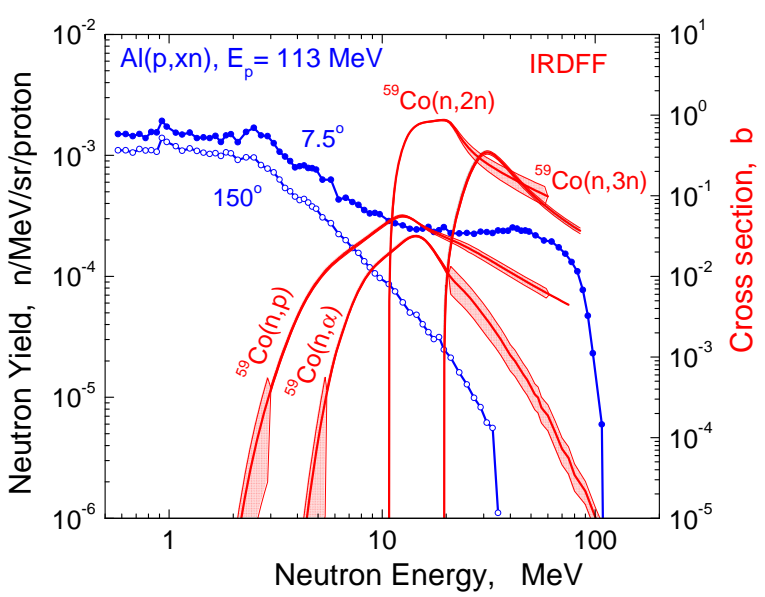

Figure 2. $\mathrm{p}(113 \mathrm{MeV})-\mathrm{Al}$ neutron spectra [11] and IRDFF dosimetry cross sections for neutron reactions on cobalt.

The classical integral experiment would be one with a broad energy spectrum extending up to $60-100 \mathrm{MeV}$, such as 113 or $256 \mathrm{MeV}$ proton beam incident on $\mathrm{Al}$ and $\mathrm{U}$ targets [9]. In this study the foil activation measurements were performed at the Intense Pulsed Neutron Source at Argonne National Laboratory. Foil packets containing $\mathrm{Al}, \mathrm{Au}, \mathrm{Co}, \mathrm{Cu}, \mathrm{Fe}, \mathrm{In}, \mathrm{Nb}, \mathrm{Ni}, \mathrm{Ti}$, and $\mathrm{Zr}$ were irradiated at a set of angels between $0^{\circ}$ and $150^{\circ}$. It is worthwhile to note that the foil activation targets, proton beam energies, and angles were chosen to match measurements that were performed separately at the
Meson Physics Facility at Los Alamos National Laboratory $[10,11]$, where the time of flight technique was used to determine the neutron energy spectra with an accuracy of $\sim 10 \%$. For illustration, Figure 2 shows $\mathrm{Al}(\mathrm{p}, \mathrm{xn})$ spectra measured at $30^{\circ}$ and $150^{\circ}$ and the IRDFF cross sections for ${ }^{59} \mathrm{Co}$ sensitive to 10 to $100 \mathrm{MeV}$ neutrons.

\subsection{Medium energies $(\approx 5-50 \mathrm{MeV})$}

The deuteron break-up reaction on light nuclear targets $\mathrm{Be}, \mathrm{C}$ or $\mathrm{Li}$ is often used to generate an intensive neutron field peaked at half of the deuteron acceleration energy [12-15]. For example, ORNL [12,15] and ANL [14] employed a d-Be source for the integral activation cross section testing program. The neutron energy spectra up to energy 11 and $45 \mathrm{MeV}$ were produced by 7 and $40 \mathrm{MeV}$ deuterons as shown in Figure 3. They were characterized by TOF technique within uncertainties $4 \%$ and $10-15 \%$. The activities of 10 and 27 activation reactions products were measured and used to test evaluated data files available at that time (ENDF/B-IV and VI).

The CRP plans to revisit available d-Be source measurements to use them for validation of IRDFF. New measurements in the well characterized broad energy fields are also encouraged.

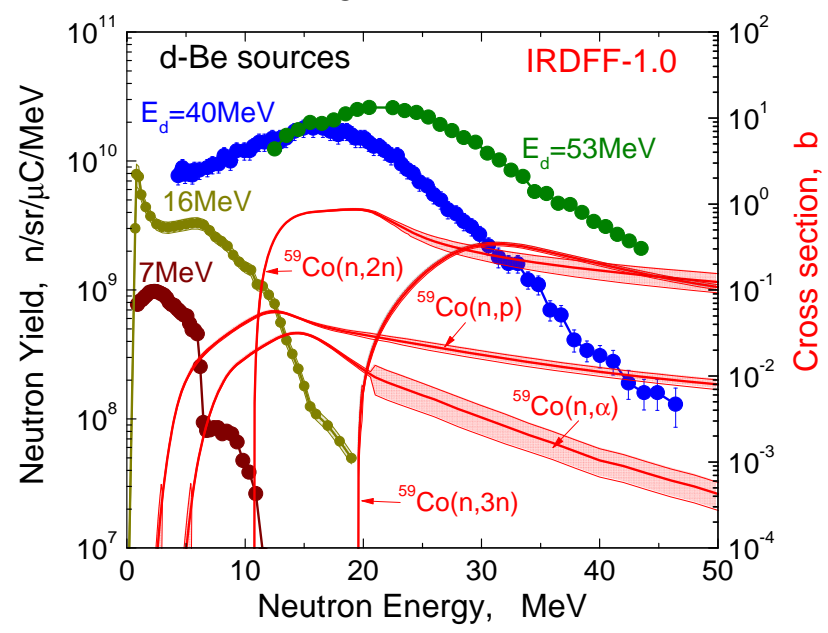

Figure 3. d-Be neutron spectra at various deuteron energies [12-15] and IRDFF dosimetry cross sections for cobalt.

\subsection{Fusion energies $(\approx 14.0 \mathrm{MeV})$}

Fusion is one of the main applications for the IRDFF library. The benchmarking in the D-T fields that have a $14 \mathrm{MeV}$ fusion peak and tail of the scattered low energy neutrons will be an important task.

During the last decades such an objective was also pursued in the validation of the European Activation File (EAF) [16] used for the fusion activation analysis. For this purpose the authors collected D-T and higher energy neutron spectra and relevant activation reaction rates. The validation of EAF was done in terms of calculated to experimental $(\mathrm{C} / \mathrm{E})$ induced activities that are a function of incident spectra and irradiation time. Figure 4 shows the typical spectra at FNS (Tokai-mura), FNG (Frascati) and TUD (Dresden) facilities and cross sec- 
tions for two dosimetric reactions ${ }^{139} \mathrm{La}(\mathrm{n}, \gamma)$ and ${ }^{89} \mathrm{Y}(\mathrm{n}, 2 \mathrm{n})$ sensitive to $\mathrm{keV}$ - or $14 \mathrm{MeV}$-neutrons. These reaction rates were measured with uncertainties $6.3 \%$ and $1.0 \%$ (see ref. in [16]).

The validation database of EAF comprises many activation reactions, however only a few of them are dosimetry reactions. This means that additional new measurements in D-T fields have to be performed to test the quality of IRDFF for fusion application.

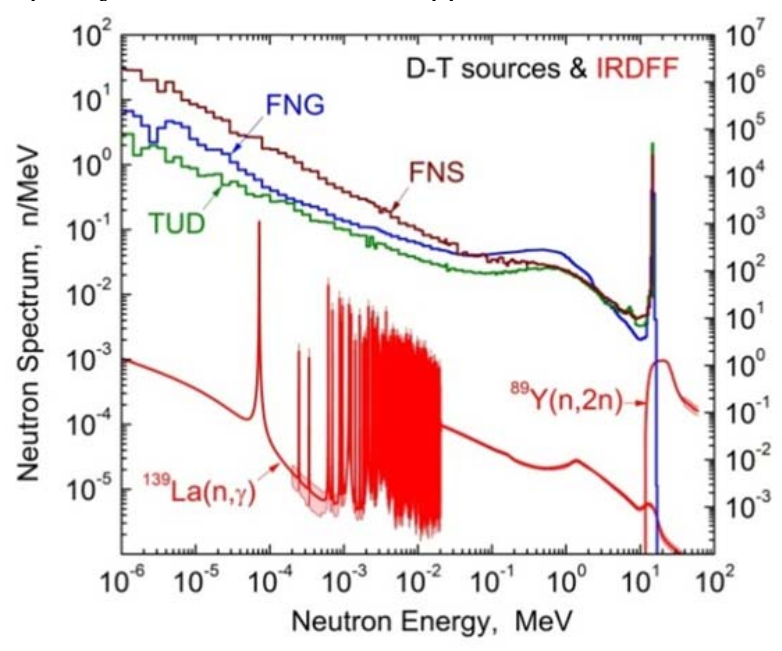

Figure 4. D-T neutron spectra collected for validation of EAF [16] and IRDFF relevant cross sections.

\section{4. $M A C S(\approx 5-500 \mathrm{keV})$}

Maxwellian averaged neutron capture reaction cross sections (MACS) were systematically measured for astrophysics applications and were collected in the EXFOR and Kadonis [17] databases. To simulate the quasi-stellar neutron spectra with different temperatures $k T=25-100 \mathrm{keV}, \mathrm{MeV}$-proton beams and thick ${ }^{7} \mathrm{Li},{ }^{3} \mathrm{H}$ or ${ }^{18} \mathrm{O}$ targets were selected. For illustration, three such spectra and the ${ }^{58} \mathrm{Fe}(\mathrm{n}, \gamma)$ reaction cross section from IRDFF are displayed in Figure 5. The MACS cross section for this reaction was measured as $13.1 \mathrm{mb} \pm 4.6 \%$ [18] whereas IRDFF predicts a higher value and larger uncertainty $16.7 \mathrm{mb} \pm 16 \%$ [2].

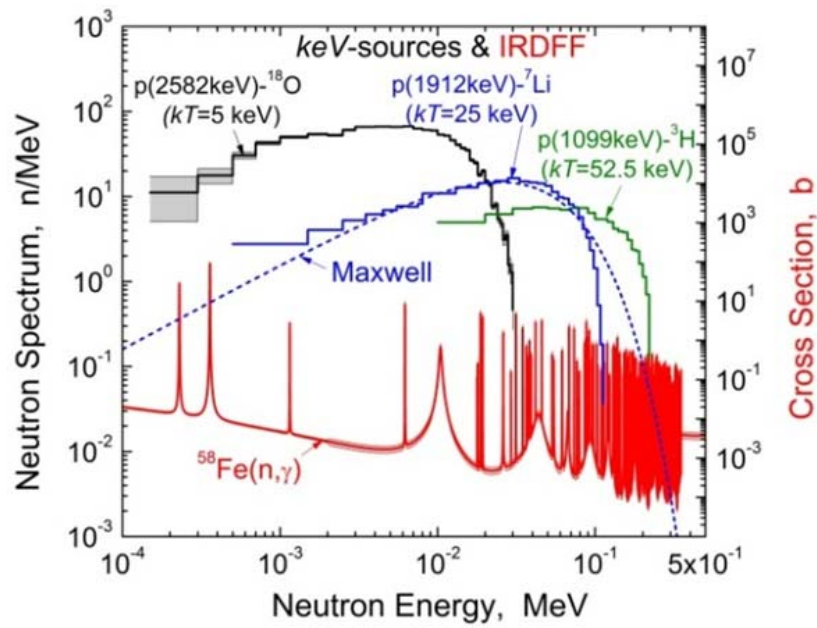

Figure 5. $\quad \mathrm{p}^{18}{ }^{18} \mathrm{O},-{ }^{\prime} \mathrm{Li}$ and $-{ }^{3} \mathrm{H}$ neutron spectra [18] and IRDFF cross section for ${ }^{58} \mathrm{Fe}(\mathrm{n}, \gamma)$ reaction.
This example shows the usefulness of already existing MACS data as well as the perceptiveness of new measurements with broad keV-neutron spectra for benchmarking of $(n, \gamma)$ and possibly $(n, p)$ or $(n, \alpha)$ reactions from IRDFF.

\section{Validation of IRDFF in the fission spectra}

The dosimetry of neutron fluxes in fission facilities remains important application for IRDFF. Its preliminary validation has been done in the well characterized neutron fields: ${ }^{252} \mathrm{Cf}$ (s.f.), thermal Maxwellian and $1 / \mathrm{E}$ slowing down spectra [2]. The predecessor of the present library, IRDF-2002, was also tested in two reference fields: Annular Core Research Reactor and an advanced fast burst Godiva type reactor (SPR-III) [1].

In the course of the CRP the existing results from the most reliable reactor benchmarks will be used for the IRDFF validation after performing careful analysis when necessary and possible. For this a short survey of two numerical databases, IRDF-2002 [1] and International Criticality Safety Benchmark Evaluation Project (ICSBEP) [18], is given here. New measurements at reactor facilities will also be very valuable.

\subsection{IRDF-2002 collection of neutron spectra}

IRDF-2002 has a collection of the reproducible reference neutron fields in the fission systems $[1,20]$. These are spherical source assemblies located inside the graphite column of reactors: Intermediate-energy Standard Neutron Field (ISNF) in Gaithersburg, MD, Coupled Fast Reactivity Measuring Facility (CFRMF) in Idaho, thermal-fast coupled field $\left(\sum-\sum\right)$ in Belgium, UK and Romania; and central hole in the fast research reactor YAYOI in Tokyo.

These spectra, shown in Figure 6, were specified by transport calculations and verified by different types of measurements in the proper energy ranges [19] (IRDF-2002 currently lacks covariance information). The facilities were used to measure many spectrum averaged dosimetry reaction cross sections (available now in EXFOR and literature) with accuracy $2-10 \%$.

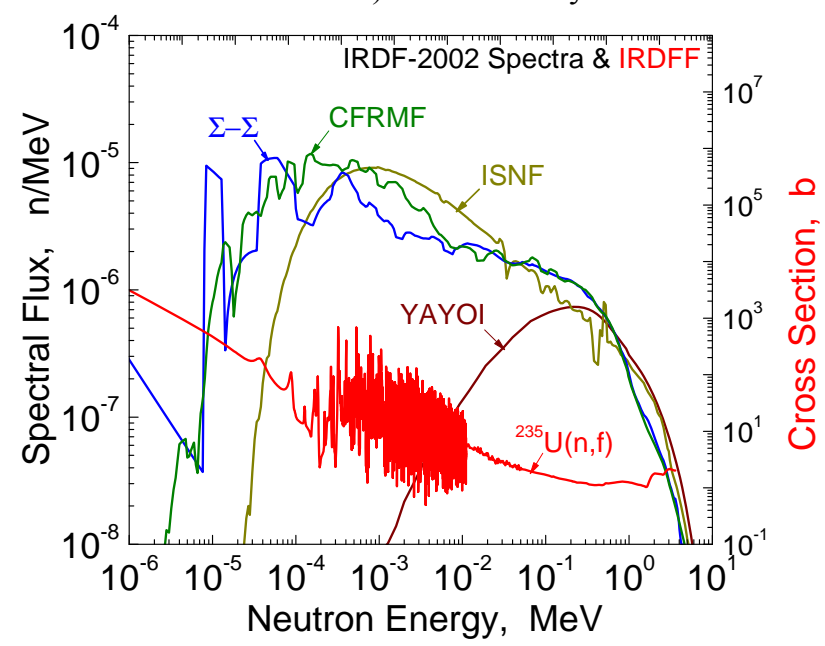

Figure 6. Reactor reference neutron spectra in IRDF-2002 [1]. 


\subsection{Critical assemblies database ICSBEP}

ICSBEP involves well documented critical benchmark cases for easy use of these data to validate calculation tools and cross-section libraries [19].

There are several assemblies (Flattop, Jezebel, Godiva, Big-Ten and Thor) where many activation rates for dosimetry reactions were measured [21]. The data are available for the $(\mathrm{n}, \mathrm{f})$ reaction on ${ }^{232} \mathrm{Th},{ }^{233,238} \mathrm{U},{ }^{237} \mathrm{~Np}$, ${ }^{239} \mathrm{Pu}$ and also for $\left.(\mathrm{n}, \gamma),(\mathrm{n}, \alpha),(\mathrm{n}, \mathrm{p}),(\mathrm{n}, 2 \mathrm{n}),(\mathrm{n}, \mathrm{n})\right)$ reactions on many stable elements included in IRDFF. The neutron spectra in these assemblies, Figure 7, are best determined by Monte Carlo simulations due to their relatively simple geometry and composition. In view of the modest neutron down scattering in some assemblies, the open issues on the prompt fission neutron spectra shape below 0.5 and above $8-10 \mathrm{MeV}$ could be tested by the low and high threshold dosimetry reactions with small uncertainties, such as standard ${ }^{197} \mathrm{Au}(\mathrm{n}, \gamma)$ and ${ }^{27} \mathrm{Al}(\mathrm{n}, \mathrm{p})$ or ${ }^{27} \mathrm{Al}(\mathrm{n}, \alpha)$. The CRP will also involve re-evaluating the LANL measured data in critical assemblies to be based on modern standards.

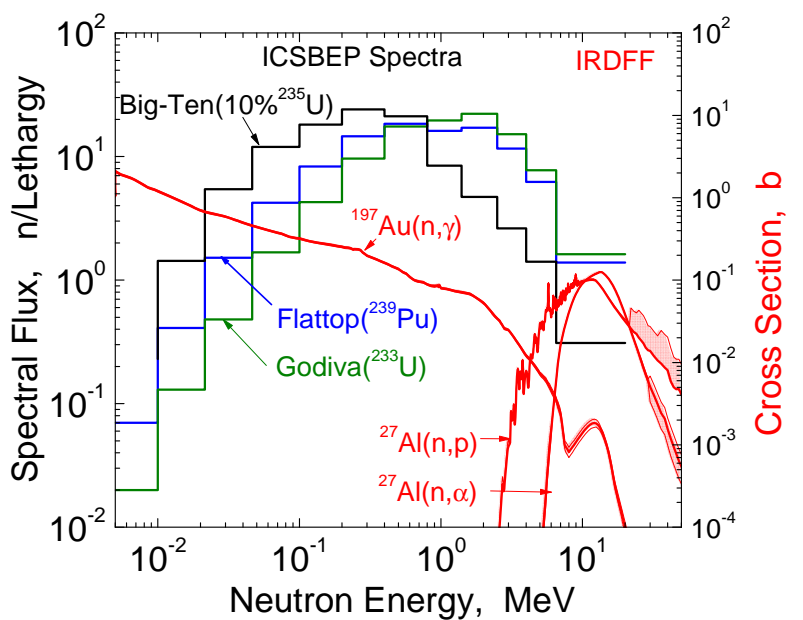

Figure 7. Critical assembly's neutron spectra from ICSBEP [19] and IRDFF cross sections.

\section{Conclusion}

An overview of the integral and differential experiments that are valuable to use for validation of the dosimetry library IRDFF is given. The quasi-monoenergetic and broad energy sources driven by accelerators and the spectra created by reactors and critical assemblies cover the energy range from 60-100 $\mathrm{MeV}$ down to thermal. The new CRP under the auspices of the IAEA will strive to initiate new measurements and collect existing information suitable for validation and testing of IRDFF.

\section{References}

[1] International Reactor Dosimetry File 2002 (IRDF-2002), Tech. Report Ser. 452, IAEA, (2006).

[2] R. Capote, K. Zolotarev, V. Pronyaev, A. Trkov, Updating and extending the IRDF-2002 Dosimetry Library, J. of ASTM Intern. 9 (2012), pp. 197-209.
[3] A. Trkov, L.R. Greenwood, and S.P. Simakov, Testing and Improving the International Reactor Dosimetry and Fusion File (IRDFF), Summary Report INDC(NDS)-0639, (2013); available on https://www-nds.iaea.org/IRDFFtest/.

[4] TENDL-2010 nuclear data library, available on: http://www.talys.eu/tendl-2010.

[5] Standard Guide for Benchmark Testing of Reactor Dosimetry in Standard and Reference Neutron Fields, ASTM Standard E2005-10.

[6] S.P. Simakov and F. Käppeler, Consultants' Meeting on Neutron Sources Spectra for EXFOR, Summary Report INDC(NDS)-0590, (2011).

[7] J.-Ch. Sublet, L.W. Packer, J. Kopecky, R.A. Forrest, A.J. Koning, D.A. Rochman, The European Activation File: EAF-2010 neutron-induced cross section library, Report CCFE-R (10) 05, Culham.

[8] N. Nakao, Y. Uwamino, T. Nakamura, T. Shibata, N. Nakanishi, M. Takada, E. Kim, and T. Kurosawa, Development of a quasi-monoenergetic neutron field using the ${ }^{7} \mathrm{Li}(\mathrm{p}, \mathrm{n}){ }^{7} \mathrm{Be}$ reaction in the 70-210 MeV energy range at RIKEN, NIM A420 (1999), pp. 218-231.

[9] L.R. Greenwood, Integral Testing of Spallation Cross Sections for Neutron Dosimetry at 113 and $256 \mathrm{MeV}$, Reactor Dos., ASTM STP 1398, (2000).

[10] M.M. Meier, C.A. Goulding, G.L. Morgan, and J.L. Ullmann, Differential neutron production cross sections and neutron yields from stopping-length Targets for 256-MeV protons, Nucl. Sci. Eng. 104(1990), pp. 339-363.

[11] M.M. Meier, D.A. Clark, C.A. Goulding, J.B. McClelland, G.L. Morgan, and C.E. Moss, Differential neutron production cross sections and neutron yields from stopping-length targets for 113-MeV protons, Nucl. Sci. Eng. 102(1989), pp. 310-321.

[12] L.R. Greenwood, R.R. Heinrich, M.J. Saltmarsh, and C.B. Fulmer, Integral tests of neutron activation cross sections in a ${ }^{9} \mathrm{Be}(\mathrm{d}, \mathrm{n})$ field at $\mathrm{E}_{\mathrm{d}}=$ $40 \mathrm{MeV}$, Nucl. Sci. Eng. 72 (1979), pp. 175-190.

[13] M.J. Saltmarsh, C.A. Ludemann, C.B. Fulmer, and R.C. Styles, Characteristics of an intense neutron source based on the Be reaction, Nucl. Ins. Meth. 145 (1977), pp. 81-90.

[14] J.W. Meadows, D.L. Smith, L.R. Greenwood, R.C. Haight, Y. Ikeda, and C. Konno, Measurement of fast-neutron activation cross sections for $\mathrm{Cu}, \mathrm{Eu}$, $\mathrm{Hf}, \mathrm{Fe}, \mathrm{Ni}, \mathrm{Tb}$ and $\mathrm{Ti}$ at 10.0 and $14.7 \mathrm{MeV}$ and for the $\mathrm{Be}(\mathrm{d}, \mathrm{n})$ thick-target spectrum, Ann. Nucl. Energy 23 (1996), pp. 877-899.

[15] S.M. Qaim, d/Be neutron fields and their applications in nuclear reaction cross-section studies (Review), Report IAEA-TECDOC-410 (1987), p. 90.

[16] R.A. Forrest, J. Kopecky, M. Pillon, A. Klix, S.P. Simakov, J-Ch. Sublet, P. Bém, M. Honusek, and E. Šimecková, Validation of EASY-2007 using integral measurements, Report UKAEA FUS 547, Culham, 2008. 
[17]Z.Y. Bao, H. Beer, F. Käppeler, F. Voss, K. Wisshak, and T. Rausher, Neutron cross sections for nucleosynthesis studies, Atomic Data and Nuclear Data Tables 76 (2000), pp. 70-154.

[18] M. Heil, F. Käppeler, E. Uberseder, R. Gallino, and M. Pignatari, Neutron capture cross sections for the weak $s$ process in massive stars, Phys. Rev. C 77, 015808 (2008).
[19] International Criticality Safety Benchmark Evaluation Project (ICSBEP), NEA, OECD, Paris.

[20] Neutron Cross Sections for Reactor Dosimetry, Report IAEA-TECDOC-208, v. 1-2, IAEA, (1978).

[21] S.C. Frankle and J.F. Briesmeister, Spectral measurements in critical assemblies: MCNP specifications and calculated results, Report LA-13675, (1999). 